\title{
La Succession Foncière de la Femme Burundaise Face à la Coutume
}

\section{Anaclet Nzohabonayo, Professeur Associé}

Enseignant-chercheur et Chef de département Administration à l'École

Nationale d'Administration/ Bujumbura-Burundi

Professeur visiteur à l'Université Officielle, à l'Université Libre des Grands

lacs et à l'Institut Supérieur Pédagogique de Bukavu en République

démocratique du Congo

\section{Docteur Jean de Dieu Ndikumana,}

Enseignant-chercheur à l'École Nationale d'administration

Professeur visiteur à l'Université lumière de Bujumbura et à Bujumbura

International University, Congo

Doi:10.19044/esj.2020.v16n17p224 URL:http://dx.doi.org/10.19044/esj.2020.v16n17p224

\section{Résumé}

Le Burundi a massivement ratifié les instruments juridiques internationaux dédiés à la lutte contre l'exclusion de la femme. Il s'est doté des programmes et politiques de planification qui consacrent une place prépondérante à la protection des droits de la femme et à son inclusion dans le tissu économique et social. Des institutions aussi bien publiques que privées œuvrent dans l'encadrement et la promotion de la femme. Ces efforts législatifs et institutionnels offrent un environnement favorable qu'il convient de mettre à profit pour développer une législation destinée à combattre les restrictions du droit de succession foncière de la femme. Pour examiner les diverses articulations de cette réflexion, la présente étude a utilisé les méthodes de recherche documentaire pour extraire des informations en exploitant les ouvrages, les articles et divers documents disponibles sur Internet. L'analyse des conventions et déclarations internationales relatives aux droits des femmes et l'examen des politiques et outils de planification a permis de développer la problématique de cette étude. Les développements de cette réflexion sont organisés autour de trois axes. La réflexion a analysé d'abord les données de l'équation de la succession foncière de la femme au Burundi (1) pour ensuite démontrer que la coutume successorale est sous l'emprise de plusieurs facteurs (2) limitant l'effectivité du droit successorale de la femme. Enfin, l'étude soutient qu'en dépit des restrictions des droits fonciers de la femme, des prémices d'un élargissement de ces droits existent (3). 
Mots-clés : Coutume, Burundi, Succession foncière, Femme

\title{
Woman's Land Succession in Burundian Custom
}

\section{Anaclet Nzohabonayo, Professeur Associé}

Enseignant-chercheur et Chef de département Administration à l'École

Nationale d'Administration/ Bujumbura-Burundi

Professeur visiteur à l'Université Officielle, à l'Université Libre des Grands

lacs et à l'Institut Supérieur Pédagogique de Bukavu en République

démocratique du Congo

\section{Docteur Jean de Dieu Ndikumana,}

Enseignant-chercheur à l'École Nationale d'administration

Professeur visiteur à l'Université lumière de Bujumbura et à Bujumbura

International University, Congo

\begin{abstract}
Burundi has embraced international legal instruments against women's discrimination. It also has adopted various programs and policy incorporating women's right protection. Many institutions have been created and dedicated to promoting its inclusion into the socio economic country life. We argue that all those factors constitute a favorable trend that should be used to develop legislation on the woman's inheritance of land. The latter is consolidating its social base and its integration into decision-making spheres. This gradual integration into the public affairs constitutes an encouraging step towards the evolution of the estate custom. To develop the topic, documentary and analytical research methods have been used. The study gathered documentation such as articles, books, legal texts as well Internet documents. We also consulted the various international agreements and declarations on the issue of women's rights and analyzed the long-term planning tools in Burundi as well as public policies relating to the gender dimension and the integration of women into decision's the spheres. The analytical methods helped us to extract necessary information for the analysis. We organized the developments around three main ideas. First of all, we examine factors influencing land succession for women in Burundi (1). Subsequently, we demonstrate that the inheritance custom is under the influence of several issues (2) such as its malicious interpretation and the slow socio-cultural evolution
\end{abstract}


of women. Although the current situation of women's land rights is not very bright, there are still signs of their possible extension (3).

Keywords: Burundi, Custom, Woman, Land inheritance

\section{Introduction}

La question de succession foncière de la femme cristallise toutes les attentions (Gatunange, 2004). La terre, en plus de sa fonction nourricière, constitue la première source de revenus pour la femme burundaise surtout de campagne (International Crisis Group, 2014). L'accès à la terre devient alors une question de survie (Commission nationale indépendante des droits de l'Homme, 2013) et la précarité des droits successoraux de la femme burundaise constitue un frein au développement économique. Aujourd'hui, la criminalité foncière figure en bonne position parmi les sources d'insécurité au Burundi (Ministère de la Sécurité publique du Burundi, 2020). Loin du grand public, les instances judiciaire et sécuritaire en connaissent les assauts continuels. Les mêmes conflits occupent plus de $90 \%$ des litiges pendant devant les juridictions burundaises (International Crisis Group, 2014).

Le Burundi est l'un des pays qui connaît la croissance démographique la plus élevée en Afrique et au monde. Parti d'une densité de 310 habitants par $\mathrm{km}^{2}$ en 2008, (Recensement général, 2008) elle a été estimée à 412 en 2017 et, il est prévu qu'en 2020 cette densité sera de 432 habitants par km² (Index mundi). Actuellement le Burundi est en troisième position parmi les densités les plus élevées en Afrique après le Rwanda et le Maurice (Index mundi année ?). Ceci signifie que la question d'accès à la terre est très épineuse, car elle devient un bien rare. Il résulte de cette carence que les conflits fonciers sont au premier rang des litiges soumis aux juridictions et que la criminalité foncière est la première source d'insécurité dans certaines contrées du pays.

Plus de $70 \%$ de la main-d'œuvre travaillent dans le secteur de l'agriculture et de l'élevage. Au sein de cette force ouvrière, les femmes constituent plus de $60 \%$. Les études ont démontré que dans les ménages burundais, les femmes s'occupent de nourrir leurs familles. Par ailleurs, elles sont de plus en plus nombreuses à être des mères célibataires et des veuves (Recensement général de la population et de l'habitat, 2008).

En dépit d'une civilisation juridique mondiale contre l'exclusion de la femme à laquelle le Burundi a souscrit, la discrimination persiste et le droit de succession foncière de la femme connaît de sérieuses limites. Il y a eu un courant doctrinal au Burundi qui a interprété la coutume burundaise comme légitimant la discrimination de la femme dans la succession foncière. La lecture actuelle de la coutume sur la succession foncière de la femme soutient que celle-ci reçoit «igiseke » (igiseke= une portion de terre attribuée à une fille mariée par ses parents) sur la terre de ses défunts parents. Cependant, une 
analyse profonde permet de démontrer qu'une fausse interprétation de cette coutume l'a détournée de son sens original dans le dessein de légitimer les intérêts des descendants mâles que la société traditionnelle burundaise a toujours considérés comme supérieurs à la descendance féminine.

De notre avis, la coutume burundaise ne contient rien de discriminatoire dans sa philosophie. Plus concrètement, cette étude postule qu'il n'y a jamais eu une coutume qui a prêché la discrimination contre la femme en matière de succession foncière. C'est son interprétation qui a créé des restrictions du droit de succession de la femme. Les mentalités masculines restent cantonnées dans le pré carré de la domination qui se ressource dans le système patriarcal. Les causes de persistance de cette interprétation erronée, dont la loi a implicitement décrété la désuétude sans succès, ont des fondements historique, socioculturel, économique, démographique et politique.

Ces quelques données du problème confirment la nécessité de prendre au sérieux la question de la succession foncière de la femme. L'urgence de cette question justifie le besoin de l'appréhender dans son évolution et proposer des réponses à la dimension des dégâts qu'elle entraîne (Kohlhagen 2009).

Pour traiter ces diverses articulations du thème, l'étude utilise les méthodes de recherche documentaire et analytique. La recherche documentaire a permis d'exploiter les livres, les articles ainsi que divers documents disponibles sur Internet pour y tirer des informations nécessaires pour cette analyse. Cette méthodologie a conduit à l'exploitation des diverses conventions et déclarations internationales sur la question de droit des femmes et analysé les outils de planification à long terme au Burundi ainsi que les politiques publiques relatives à la dimension genre et à l'insertion de la femme dans les sphères de décision. Cette documentation diversifiée a permis de mener des analyses pour développer les arguments et les articulations de cette réflexion. Les développements de cette réflexion sont organisés autour de trois axes. Pour aborder cette thématique de succession foncière de la femme dans la coutume burundaise, l'étude expose d'abord les données de l'équation de la succession foncière de la femme au Burundi (1). Cette démarche permettra d'identifier les divers statuts de femmes à vocation successorale (1.1) pour analyser les droits fonciers de la femme dans la coutume burundaise (1.2). Sous cette rubrique l'analyse portera aussi sur les changements de statuts de la terre qui affectent les droits fonciers de la femme (1.3). Par la suite, les développements démontrent que la coutume successorale est sous l'emprise de plusieurs facteurs (2) dont notamment la manipulation caractérisée par son interprétation malicieuse (2.1) et la lente évolution socioculturelle de la femme (2.2). Si la situation actuelle des droits fonciers de la femme est peu reluisante, il existe tout de même des prémices d'un possible élargissement de ces droits 
(3). Il existe un cadre légal prévoyant l'égalité des droits (3.1) et un cadre politique et institutionnel progressiste sur la question successorale de la femme (3.2).

\section{Succession foncière de la femme : les données de l'équation}

$\mathrm{Au}$ sein de la population féminine burundaise, on distingue plusieurs catégories de femmes avec des statuts à vocation successorale très diversifiés selon la coutume. Mais la question de la succession foncière de la femme a aussi évolué au gré des changements qui ont affecté le statut de la terre.

\subsection{Des statuts de femmes à vocation successorale}

Lorsqu'on parle de la question successorale de la femme en matière foncière, le premier exercice est celui de savoir de quelle femme il s'agit.

D'une part, la question concerne spécifiquement les femmes qui vivent en milieu rural et dont leurs activités principales sont l'élevage et l'agriculture. Pour cette catégorie, les droits de succession varient selon le statut matrimonial et de descendance familiale de la femme. Pour ce qui est du statut matrimonial de la femme, il s'agit d'abord de savoir si la femme est mariée, veuve avec ou sans enfant, divorcée ou célibataire endurcie. L'appartenance à l'une de ces catégories influe sur sa succession foncière. Ensuite, en parlant de la condition de descendance, il est question de savoir si la femme est issue d'une descendance féminine autrement si dans sa famille, il y a des garçons ou non.

D'autre part, une autre donnée du problème est que la succession foncière prend des proportions différentes en fonction de la couche féminine considérée. Les droits de succession des femmes rurales diffèrent de ceux des femmes en milieux urbains. Il s'agit alors d'une même coutume que l'on doit interpréter pour en dériver les droits qui reviennent à chacune des catégories de femmes susmentionnées. Il faut aussi signaler que les droits de la femme sont déterminés par rapport à sa famille d'alliance; c'est-à-dire là où elle est mariée.

Une autre donnée fondamentale dans cette problématique de succession foncière concerne la nature de la propriété foncière sur laquelle les droits de succession de la femme portent. Dans le paysage du foncier familial, deux catégories coexistent. C'est d'abord la propriété familiale à proprement parler; c'est-à-dire celle où est né leur ancêtre. Autrement dit, c'est celle dont les ascendants de la femme ont hérité de leurs propres parents.

Ensuite, il y a le foncier acheté par les parents défunts de leur vivant. Selon qu'il s'agisse de l'un ou l'autre foncier, les droits fonciers successoraux de la femme varient. 


\subsection{Les droits fonciers de la femme dans la coutume}

La femme mariée recevait une portion de terre qu'elle cultivait du vivant de ses parents. Ceux-ci avaient le devoir de continuer à traiter la femme mariée comme membre à part entière de la famille. Comme ils ne pouvaient pas à chaque fois lui apporter des cadeaux dans sa belle famille, cette portion de terre permettrait de garder le lien avec sa famille d'origine. C'était aussi une façon de combattre l'exclusion de la fille dans le partage de la richesse familiale qui était basée sur l'exploitation agricole.

À l'ouverture de la succession, elle recevait, et c'est toujours le cas dans certaines situations et parties du territoire, une portion de terre sous forme d'usufruit (igiseke) dont le droit expire à son décès. Si la femme ne pouvait pas transmettre sa portion à sa descendance, c'était dans la logique de la coutume qui interdisait la transmission du patrimoine foncier dans un autre clan (Ncutinamagara, 1980).

Jusque là, la limite de ce droit successoral de la femme touchait aussi celui du descendant mâle qui ne pouvait transmettre, ni aliéner de quelque manière que ce soit, la propriété de la terre à une personne étrangère à la lignée. En toute hypothèse, la terre restait donc dans la famille, et il était difficile de soutenir l'existence d'une discrimination coutumière du moment où la fille tout comme le garçon ne disposait que des droits d'usufruit sur la terre de leurs ancêtres. Donc, dans sa famille biologique, la coutume permettait à chaque fille l'accès à la terre ancestrale.

Dans sa famille d'alliance, les droits successoraux appartiennent au mari. Ils se transmettent à la femme avec la mort de son conjoint, mais de manière très nuancée. Lorsque la femme veuve avait des enfants, elle prenait la place de son mari défunt et, sous réserve de ne pas y ajouter d'autres enfants issus d'un autre homme, elle jouissait du statut d'administratrice des biens laissés par son défunt mari. La femme veuve sans enfant se trouverait ainsi dans une situation précaire. Ses droits dépendaient largement du bon vouloir des membres de la famille du mari. Ils pouvaient lui proposer un autre mari, la laisser dans la propriété de son mari ou tout simplement la forcer d'une manière ou d'une autre, à quitter les lieux.

Les filles issues d'une descendance exclusivement féminine ne pouvaient pas hériter de leurs parents. On leur préférait leurs oncles paternels qui partageaient en pleine propriété cette terre au détriment des descendants féminins. Actuellement, la situation des filles issues d'une descendance féminine a évolué et elles peuvent hériter de leurs parents. Auparavant, la propriété revenait aux oncles paternels. Mais cette solution était devenue injustifiable compte tenu du principe d'égalité de tous les enfants contenus dans les instruments juridiques internationaux que le Burundi a ratifiés [art 25 (2) de la Déclaration universelle des droits de l'homme]. Ainsi, quand elles n'ont pas de frères, les filles héritent de plein droit de la propriété familiale, 
comme si elles étaient des garçons. À l'analyse de la jurisprudence, on observe une dynamique en faveur de l'égalité entre les filles et les garçons pour ce qui concerne par exemple la propriété «itongo ryumuheto» (qui n’est pas familiale).

La femme divorcée ou veuve qui retourne chez ses parents, ou la fille célibataire endurcie ne pouvaient selon les cas, être traitées sur un même pied d'égalité que les garçons. Ces catégories de femmes héritent de la propriété foncière au gré des humeurs de leurs frères. Dans certains cas, ces femmes héritent d'une portion de terre inférieure à celle de leurs frères. Dans d'autres situations, les frères consentent à un partage égal. Les pratiques varient selon les régions d'un cas à l'autre.

\subsection{Des droits successoraux soumis aux changements du statut de la terre}

Le sort des droits successoraux de la femme a évolué au gré des changements du statut de la terre au Burundi. À l'époque monarchique, et ceci en dépit de la colonisation, la terre au Burundi avait un statut collectif. Mais quatre ans après l'accession à l'indépendance (1966), la terre est devenue un bien privé susceptible d'appropriation.

\subsection{Sort des droits successoraux de la femme à l'époque monarchique}

À l'époque monarchique, la terre appartenait au Roi qui était seul propriétaire (lettre de politique foncière du 15 octobre 2008). Les structures administratives du royaume dont les chefferies avaient le mandat de distribuer des terres aux chefs de clans. La terre avait ainsi un caractère collectif. La terre était un bien attaché à un lignage, c'est-à-dire à un groupe de parenté, et sa transmission se faisait exclusivement par voie patrilinéaire. Le père laisse la terre à son fils qui le transmettra à son fils. Le droit de celui-ci se limitait à poser des actes de gestion et de conservation du bien foncier dans le lignage sans en avoir le droit de l'aliéner. Autrement dit, la propriété passerait dans une famille étrangère.

Cette logique lignagère ne semble pas consacrer l'exclusion de la femme qui reste après tout dans son lignage et qui conserve les mêmes droits d'accès. Le fils en question était plus l'administrateur (voire le gardien) que le dépositaire. Du moment que la terre avait un statut collectif et appartenait au clan, aucun descendant mâle ou femelle ne pouvait en transférer la propriété ou s'en approprier. La logique foncière familiale était de conserver le bien foncier dans la famille. À ce moment, la femme mariée avait, tout comme son frère, le droit de cultiver une portion de la terre familiale que ses parents lui indiquaient. C'est cette portion qui prend la dénomination de "Igiseke» signifiant une portion de terre. 
À sa mort, la portion de terre qu'elle exploitait revenait à la famille et ainsi le clan avait toujours la maîtrise de la terre comme le veut la logique collectiviste de la terre. On a dérivé de cette pratique ce qui est devenu une coutume d'alors en matière de succession foncière de la femme au Burundi. Cette coutume veut que la femme mariée reçoive le "igiseke" qui reviendra aux descendants mâles à sa mort.

\subsubsection{Sort des droits successoraux de la femme après l'abolition de la monarchie}

L'ébranlement de l'administration monarchique survenu avec la destitution du Mwami (le monarque) le 28 novembre 1966 et des chefs comme anciennes autorités foncières a remis en question la gestion foncière traditionnelle. L'abolition de l'«Ubugererwa (le servage aboli le 30 juin 1977) » et la poussée de la mondialisation ont finalement sonné le glas du régime traditionnel de gestion foncière. Le statut collectif s'est estompé et a cédé la place au statut privé de la terre. Celle-ci est devenue désormais susceptible d'appropriation individuelle et chacun pouvait revendiquer une portion de la succession.

Ayant acquis le statut de propriété individuelle, la terre pouvait désormais faire objet de marchandisation dont la conséquence est de transférer la propriété dans une autre famille. La présente étude considère que c'est le passage à cette appropriation individuelle qui a entraîné le début des pratiques discriminatoires que la gent masculine a légitimé à tort par la coutume.

Cette réflexion soutient que le postulat de la coutume burundaise et même sa finalité n'ont jamais été de précariser le droit successoral de la femme. Autant celle-ci ne pouvait pas vendre, autant son frère ne pouvait pas non plus transférer la propriété. L'étendue du droit d'accès à la terre était identique chez tous les membres de la famille sans distinction de genre.

Une analyse de ce qui précède permet de réfuter l'argument réducteur tenu par les défenseurs de la prédominance de la masculinité qui examinent l'«igiseke» comme une petite portion de terre pour s'en servir dans la restriction du droit successoral qui revient à la femme. Celle-ci était dans son foyer où son mari avait les droits similaires à ceux dont disposaient ses beaux frères dans leurs familles. Il y avait donc une sorte de compensation naturelle qui rendait inopportun le débat sur la taille de la portion que la femme recevait.

Le tournant fatidique pour le droit successoral de la femme commence à se dessiner avec l'importance économique grandissante que la terre a prise au Burundi pendant la période post-coloniale. Le pays a embrassé l'économie libérale qui fait du droit de propriété son fondement essentiel. La terre est devenue alors une source de convoitise et de richesse individuelle. Progressivement, on quittait une situation d'égalité où toute la fratrie utilisait 
la terre dans le respect de son statut collectif pour instaurer une discrimination en défaveur de la gent féminine.

\section{Une coutume successorale sous l'emprise de la manipulation et de la lente évolution socioculturelle de la femme}

\subsection{Des droits successoraux sous l'emprise d'une interprétation malicieuse de la coutume}

Selon les pratiques répandues de la coutume successorale, la femme rurale hérite d'une parcelle de terre qu'elle cultive. À sa mort, la parcelle reste la propriété de la famille. Dans la logique de gestion coutumière de la terre, on devait la conserver dans la lignée. La descendance masculine en assurait l'administration et l'exploitation sans droit de l'aliéner sans le consentement du clan. Mais avec l'évolution et l'importance de la terre, la gent masculine a tordu le cou à cette coutume en usurpant le droit de propriété sur la terre (Kohlhagen, 2009).

L'analyse réfute les thèses conservatrices qui légitiment cette restriction des droits de succession foncière par la coutume. La gent masculine, profitant de sa prédominance dans l'organisation socioéconomique du pays, mais aussi du privilège de masculinité (Gatunange, 2015), a attribué à la coutume burundaise une intention qu'elle n'a jamais eue : démembrer le droit successoral de la femme mariée pour le réduire à l'usufruit. Les descendants mâles disposent de la pleine propriété sur leurs portions alors que les femelles héritent de l'usufruit (Action Aid, Rapport parallèle au comité des Nations unies des droits de l'Homme Burundi, 2014).

Cette analyse postule que la coutume successorale burundaise n'a jamais prêché la supériorité de la masculinité sur la féminité. Il existe aussi une littérature plutôt activiste que scientifique qui attribue la discrimination successorale de la femme à la coutume (Ncutinamagara, 1980). Cette conception est toute aussi fausse que partisane et contribue à alimenter la surenchère. L'économie d'analyse doctrinale scientifique des juristes burundais sur cette question a permis l'extension de cette lecture -que cette étude considère erronée- de la coutume en matière de succession de la femme. Rappelons-le, la philosophie de la coutume burundaise reposait sur le principe cardinal de conservation de la terre dans la lignée. Ce principe était aussi impératif pour les descendants mâles que les descendants femelles. Le chef de famille et/ou de clan n'avait pas le droit de déplacer la terre (aliéner/transférer) à plus forte raison ses héritiers. Tout le monde était dans cette logique de droit d'usufruit sur la terre des ancêtres dont il n'était pas dépositaire. C'est l'interprétation malicieuse qui a tordu le cou à une coutume qui jusque là véhiculait les valeurs d'égalité. Une des preuves de cet argument est qu'aujourd'hui en milieu urbain, le consensus d'égalité homme-femme 
commence à émerger et les enfants héritent équitablement les propriétés achetées par leurs parents.

La lecture qui semble plus réaliste est celle qui considère que la coutume burundaise prêche que le descendant mâle soit gestionnaire de la terre et pas son propriétaire, son bénéficiaire et pas son dépositaire et que tout cela doit être fait pour utiliser la terre sans compromettre la capacité des générations futures à en jouir (IUCN, 1983).

C'est ainsi que la coutume qui était conçue pour égaliser les droits des descendants du «de cujus» (du défunt) a été utilisée comme un outil d'oppression pour brimer les droits successoraux de la femme. À partir de ce moment, la coutume, faussement interprétée, et d'ailleurs on venait de la vider de tout son sens en privatisant la terre, est devenue caduque. L'homme burundais a été, et demeure, responsable de la dispersion de la terre en dehors de la famille et; c'est à notre sens une responsabilité qu'il devrait assumer et rétrocéder à ses consœurs la pleine propriété de leur droit successoral. Le statut successoral de la femme est ainsi sous l'emprise d'une interprétation tendancieuse de la coutume qui réduit ses droits au profit d'une masculinité qui puise sa prédominance en partie dans le système patriarcal burundais et dans la position qu'occupait l'homme dans les instances de décision. Certes, la coutume a son «péché congénital»d'être orale et par conséquent moins précise que la loi écrite. Mais cette faiblesse semble largement insuffisante pour légitimer la réduction des droits fonciers successoraux de la femme.

\subsection{Des droits sous l'emprise de la lenteur de l'évolution socioculturelle de la femme}

La couche masculine a toujours cantonné la femme dans une place de second rang que la coutume ne lui a jamais attribuée. C'est plutôt l'organisation sociale et administrative qui assignait aux différentes composantes de la communauté burundaise des rôles à assumer au sein de la société.

La structure de la société burundaise de l'époque composait bien avec cette division de travail au point que la femme ne se sentait jamais rabaissée. Mais le choc entre ces valeurs de la société traditionnelle et l'occidentalisation consécutive à la colonisation a provoqué une mutation de la société burundaise. Ce processus d'européanisation a conforté la place de l'homme burundais sur la scène politique et administrative.

Cette prédominance dans les cercles décisionnels lui a permis d'accaparer l'espace social et de développer une culture de supériorité qui a légitimé pendant un temps l'écart dans l'éducation, la faible participation dans les institutions de la République, la mainmise sur l'économie familiale etc. Il s'en est suivi un rétrécissement de la vitrine d'expression pour les femmes auxquelles le choix de la résignation et du silence a été imposé. C'est cet état 
de choses qui a permis à l'homme d'imposer sa vision de la coutume et d'imprimer la lenteur de l'évolution socio-économique et culturelle de la femme.

Mais aujourd'hui, l'occidentalisation qui a fait « les honneurs » de l'homme dominant lui impose une autre démarche : celle de respect des droits de l'Homme dont les valeurs en constant apogée ont placé les hommes nostalgiques du passé à la défensive lorsqu'on en vient à la question d'accès à la terre. Il se refuse d'assumer dans les faits le discours officiel sur l'égalité sachant pertinemment que le destin sur la terre burundaise restituera la vérité. Certaines femmes préfèrent s'abandonner au sort d'une prétendue civilisation humaine dont elles subissent les affres. Pour celles-là, la mentalité est à la soumission. Elles perçoivent celles qui revendiquent un accès égal à la terre comme des auteures de la dépravation de l'ordre social foncier au Burundi.

Aussi bien parmi les hommes que les femmes, il n'est pas rare d'entendre des arguments fustigeant l'autonomisation des femmes. Pour eux, renforcer les droits économiques des femmes pourrait «perturber les rapports sociaux et le respect que les femmes doivent aux hommes ». En dépit de la persistance de ces mentalités rétrogrades et de la catégorie d'hommes qui sont restés sclérosés dans leur supériorité, supposée ou imaginaire, il existe, surtout dans les villes, une tendance dominante chez les hommes qui sont parfaitement en harmonie avec le principe d'accès égal au foncier pour tous les enfants. Il y a donc une évolution de mentalités qui s'amorce.

$\mathrm{Au}$ fait, cette réflexion soutient que la conservation de la terre dans la lignée a été compromise par l'évolution du droit de propriété qui était devenue inéluctable surtout dans les sociétés modernes où le capitalisme est devenu le mode d'organisation économique le plus dominant. La gent masculine s'est alors arrogé le droit d'aliéner la terre, transformant ainsi le droit d'usufruit qu'elle avait sur la terre en un droit en pleine propriété.

De cette appropriation indue, et de surcroit contraire à la coutume, on venait d'hypothéquer l'égalité qui a toujours caractérisé la femme et l'homme qui n'avaient tous deux que des droits d'usufruit sur la terre ancestrale. Pour légitimer les parts léonines (la part du lion) que les hommes s'attribuent dans le partage de la terre, l'opinion masculine dominante prend en otage une coutume à laquelle elle ne veut pas restituer ses fondements originaux d'égalité et d'équité.

\section{Les prémices de l'élargissement des droits fonciers successoraux de la femme}

L'évolution du cadre légal et institutionnel en cours permet d'envisager une évolution des droits fonciers successoraux en faveur de la femme au Burundi. 


\subsection{La lettre foncière}

La lettre de politique foncière adoptée en 2010 fait un bilan moins clément, mais très réaliste sur la problématique des terres au Burundi (décret $\mathrm{n}^{\circ}$ 100/72 du 26 avril 2010 portant adoption de la lettre de politique foncière au Burundi). La philosophie de cette lettre cadre avec les accords d'Arusha qui recommandaient la révision du code foncier pour répondre aux problèmes actuels de la gestion des terres (Accord d'Arusha de 2000). Cette lettre ne traite pas la question de l'accès des femmes à la terre. Cependant, elle dessine un portrait sombre de ce qu'elle qualifie de « crise foncière » (décret $\mathrm{n}^{\circ}$ 100/72 du 26 avril 2010 portant adoption de la lettre de politique foncière au Burundi) dont l'effet sur la restriction des droits de la femme suscite moins de doutes. Le panorama de difficultés de gestion de terre que dressent les rédacteurs de la lettre foncière montre la pertinence de la question d'accès au foncier au Burundi (décret $\mathrm{n}^{\circ} 100 / 72$ du 26 avril 2010 portant adoption de la lettre de politique foncière au Burundi). Dix ans après cette lettre foncière, et en dépit des améliorations, apportées, des problèmes subsistent.

Le mérite de ce document dont l'importance suscite moins de discussion est d'avoir provoqué un sursaut de prise de conscience sur la thématique foncière. Plusieurs chantiers législatifs, dont le code foncier et le code de l'environnement, sont consécutifs aux orientations de cette lettre foncière. L'amélioration des droits de la femme devait suivre. Cependant, la lettre foncière est un document-cadre dont la nature est d'orienter la prise de décisions sur les actions à mener. Pour en tirer profit, des efforts de définition des politiques pour opérationnaliser les orientations devaient suivre. Dans ce cadre, plusieurs politiques ont été mises en œuvre. Entre autres politiques, l'enregistrement foncier des terres rurales. Celui-ci devait permettre d'assurer la sécurité juridique des droits fonciers sur les terres rurales. Dans les communes pilotes, cet enregistrement a donné lieu à des certificats fonciers. Dans les autres communes, la politique a connu moins ou pas de succès. C'est au travers de cette politique que la décentralisation de la gestion foncière devait être opérée.

Les effets de la lettre foncière sont mitigés. D'abord, les ressources financières ont manqué pour mettre en place de véritables institutions au niveau des communes dotées de moyens pour atteindre les objectifs consignés dans la lettre foncière. Ensuite, la politique foncière a manqué de soutien à la base en raison d'une forte assise culturelle opposée à tout ce qui viendrait remettre en cause le mode de gestion actuelle qui place la gent masculine au sommet du cercle décisionnel. 


\subsection{Existence d'un Cadre légal prévoyant l'égalité des droits d'accès à la terre}

Le Burundi a ratifié les instruments juridiques internationaux en plus de l'adoption des différentes déclarations relatives aux droits de l'homme.

\subsubsection{Cadre conventionnel}

L'adhésion du Burundi à l'ONU a eu pour corollaire d'incorporer la Déclaration universelle des Droits de l'Homme dans son cadre légal interne. Cet instrument consacre en son article 17 le droit à la propriété pour toute personne et réaffirme que nul ne peut être arbitrairement privé de sa propriété. Dans sa philosophie législative, protéger et garantir la dignité de la femme constituent le leitmotiv de l'appareil législatif burundais. Le pays a ratifié le pacte international relatif aux droits économiques, sociaux et culturels (loi $\mathrm{n}^{\circ} 11 / 008$ du 14 mars 1990). En son article 3, ce texte consacre la jouissance de tous les droits économiques en énonçant : «Les États parties au présent Pacte s'engagent à assurer le droit égal qu'ont l'homme et la femme au bénéfice de tous les droits économiques, sociaux et culturels qui sont énumérés dans le présent Pacte » (Sebudandi \& Ndikumana, 2012).

Le Burundi est respectivement membre de la Convention sur l'Élimination de toutes les formes de Discrimination à l'égard des Femmes (CEDEF) (ratifiée le 8 janvier 1992) et du protocole additionnel à la Charte africaine des droits de l'Homme et des peuples relatifs aux droits de la femme en Afrique signé le 13 novembre 2001. Au sens de la CEDEF, «la discrimination à l'égard de la femme vise toute distinction, exclusion ou restriction fondée sur le sexe qui a pour effet ou pour but de compromettre ou de détruire la reconnaissance, la jouissance ou l'exercice par les femmes, quel que soit leur état matrimonial des droits de l'Homme et des libertés fondamentales dans le domaine politique, économique, social, culturel et civil, et dans tout autre domaine ». L'article 2 de la CEDEF engage les États parties à «poursuivre par tous les moyens appropriés et sans retard une politique tendant à éliminer la discrimination à l'égard des femmes ».

Le Burundi a également ratifié le 28 juillet 1987, la Charte africaine des droits de l'Homme et des peuples de 1981, de même que la Convention sur les droits politiques de la femme ratifiée le 31/12/1992, par le Décret-loi $\mathrm{n}^{\circ} 1 / 006 \mathrm{du} 4$ avril 1991.

Le Burundi s'est aussi aligné à la Déclaration solennelle pour l'égalité de genre en Afrique, à la Déclaration sur les problèmes et enjeux fonciers en Afrique, aux Directives volontaires pour une Gouvernance responsable des régimes fonciers applicables aux terres, aux pêches et aux forêts dans le contexte de la sécurité alimentaire nationale ainsi qu'aux Cadres et lignes directrices sur les politiques foncières en Afrique etc. 
L'adhésion à ces différentes conventions internationales et la souscription à certaines de ces déclarations ont eu pour effet l'incorporation de ces outils dans le droit positif burundais. Cette partie de la législation burundaise constitue à notre sens une volonté manifeste des autorités de la République de traiter au haut niveau également tous les citoyens sans discrimination basée sur le sexe. Le cadre légal national s'oriente, dans une certaine mesure, dans cette direction favorable au traitement inclusif des femmes dans la succession foncière. Évidemment on peut déplorer le décalage qui s'est produit entre la coutume et l'évolution sociale et économique, provoquant par là un traitement différencié sans que cela puisse être le fait de la coutume elle-même ou une volonté délibérée d'exclusion des femmes de l'héritage foncier de leurs géniteurs.

\subsubsection{Cadre légal national}

Le Burundi a manifesté, et c'est toujours le cas, son attachement à régler la question de succession foncière de la femme. Comme les développements précédents l'ont montré, il a, et continue, de poser les bases juridiques pour lutter contre la discrimination.

Les prémices qui s'inscrivent dans la dynamique de la modernisation $\mathrm{du}$ droit foncier et de son accès pour les femmes remontent de l'adoption des Accords d'Arusha en 2000 (Accord d'Arusha signé le 28 août 2000). Ce texte a consacré l'égalité de tous devant la loi et on se souviendra qu'il était signé pour guérir les plaies de la discrimination ethnique. Les parties à sa négociation en ont profité pour murir la question et poser des jalons pour combattre les formes de discrimination dirigées contre les femmes.

La Constitution burundaise de juin 2018 pose et défend le principe de l'égalité entre tous les citoyens. En son article 13, ce texte fondamental prévoit l'égalité entre tous les Burundais et la jouissance des mêmes droits par tous les citoyens sans distinction basée notamment sur le sexe (Constitution, 2018). La même constitution dispose en son article 19 que «les droits et devoirs proclamés et garantis, entre autres, par la Déclaration universelle des Droits de l'Homme, les Pactes internationaux relatifs aux droits de l'Homme, la Charte africaine des Droits de l'Homme et des peuples, la Convention sur l'élimination de toutes les formes de discrimination à l'égard des femmes et la Convention relative aux droits de l'enfant font partie intégrante de la Constitution de la République du Burundi » (Constitution, 2018). L'article 22 de la même constitution ajoute que «nul ne peut être l'objet de discrimination du fait notamment de son origine, de sa race, de son ethnie, de son sexe [...]».

La proposition de loi sur les successions, régimes matrimoniaux et libéralités qui a avorté en 2004 est l'un des exemples pour opérationnaliser la volonté de régler la question successorale de la femme (Proposition de loi de 2004). Le code foncier de 2011 abonde dans le même sens lorsqu'il énonce 
que «toute personne physique ou morale peut jouir, sans discrimination aucune [...] de tous les droits définis dans le présent code et les exercer librement, sous réserve du respect des droits d'autrui et des restrictions résultant du respect de la loi » (art 12 du code foncier révisé).

Le Code foncier, qui distingue les terres appartenant à l'État (domaniales ou à titre privé) des terres appartenant aux citoyens, reconnaît les droits de propriété relatifs aux biens fonciers : les propriétaires terriens ont le droit d'utiliser de manière exclusive leurs terres, d'en disposer librement et de les hypothéquer. Le Code foncier prévoit que les terres peuvent être enregistrées soit au nom de l'époux, soit au nom de l'épouse. On doit toutefois « noter que dans ce cas, il s'agit généralement de terres achetées en son nom propre et n'appartenant pas à la famille du mari, soit au nom des deux » (Cangelosi \& Pallas, 2014).

Au travers de ce code foncier, et la constitution de 2018, les principes d'égalité des sexes et de non-discrimination basée sur le sexe sont consacrés afin de pallier le faible accès des femmes à la terre et l'insécurité qui caractérisent leurs droits fonciers (Kotchikpa, 2017). Cela signifie que les femmes sont désormais en mesure d'acheter des terres. Le Code des Personnes et de la Famille, amendé en 1993, est également relativement favorable à l'accès des femmes à la propriété, puisqu'il prévoit la gestion commune des propriétés familiales.

D'autres cadres et documents de politique peuvent aussi servir de base et guider le gouvernement du Burundi pour oser et concrétiser sa volonté en codifiant le droit de succession pour soustraire son sort aux caprices du privilège de la masculinité qui a détourné et imposé une conception de la coutume successorale que les ancêtres ne lui ont jamais prêtée.

\subsection{Cadre politico-institutionnel}

Les droits successoraux de la femme figurent en bonne place dans diverses politiques publiques au Burundi. Des institutions en place peuvent œuvrer pour la réalisation de ces droits en dépit d'une culture à laquelle les tenants du conservatisme masculin opposent une résistance qui s'érode progressivement.

\subsubsection{Le cadre institutionnel}

Le cadre institutionnel se compose des institutions qui relèvent de la sphère étatique et d'autres qui ont un statut privé.

\subsubsection{Le cadre institutionnel inféodé à l'État}

L'institution d'Ubushingantahe a été toujours la pierre de lance de la justice de proximité au Burundi (Rodegem, 1966). Les bashingantahe (les sages) sont à l'avant-garde dans le règlement des litiges locaux. L'Abbé 
Adrien Ntabona définit le Mushingantahe comme un « homme responsable du bon ordre, de la tranquillité, de la vérité et de la paix dans son milieu. Et cela, non pas en vertu d'un pouvoir administrativement attribué, mais de par son être même, de par sa qualité de vie, que la société voulait reconnaître à sa personne en lui conférant une investiture » (Ntabona, 1985).

Investis de cette fonction de réconcilier les gens en tranchant les litiges de son voisinage, l'institution d'Ubushingantahe dispose d'un immense potentiel pour contribuer à l'évolution de la coutume en se ressourçant dans ce cadre légal et conventionnel en constante évolution (Makobero, 2001). Les Bashingantahes sont les premières autorités collinaires saisies des questions foncières avant le déclenchement du contentieux juridictionnel (article 37 de la loi communale de 2005). Les valeurs morales de paix et de justice dont ils sont imbus en font des piliers de l'organisation sociale, économique et politique capable de réconcilier les adversaires et de redresser les détracteurs de la normativité de l'ordre social (Deslaurier, 2003 (No 92)).

Mais en dépit de ses faiblesses actuelles, l'importance de cette institution s'est renouvelée avec le crédit que lui ont accordé les négociateurs de l'Accord d'Arusha (Accord d'Arusha). La présente étude estime qu'avec une volonté politique de restituer à cette institution son prestige d'antan (Laely, 1992, pp. 75-98), elle peut recouvrer sa vitalité. Des efforts dans ce sens existent, même si les résultats sont parfois en deçà des attentes (décretloi no 1/001/97 du 3 janvier 1997 et du 21 mars de la même année). Les fonctions des Bashingantahe s'accommodent mal avec un immobilisme culturel qui fige la coutume. Celle-ci doit s'adapter à l'évolution mondiale de la prise en considération des droits des femmes. Les Bashingantahe qui usent de leur sagesse ont besoin de s'imprégner des nouveaux développements au travers des sessions de perfectionnement en vue de l'acquisition de nouvelles connaissances. Ces dernières sont déterminantes pour sécuriser à la base les droits fonciers de la femme.

Le gouvernement burundais s'est doté d'un Ministère des Droits de la personne humaine, des Affaires sociales et du Genre. La protection des droits des femmes et la promotion de l'égalité font partie des innombrables missions assignées à ce ministère (Décret $\mathrm{n}^{\circ} 100 / 57$ du 4 avril 2016). Au sein de ce ministère, il existe une direction générale des droits de la personne humaine, de l'éducation à la paix et la réconciliation nationale. Pour tenir compte de la particularité de la situation des femmes au Burundi, on a consacré une direction générale à la promotion de la femme et du genre (Décret $\mathrm{n}^{\circ} 100 / 57$ du 4 avril 2016). Cette direction a pour mandat de veiller à l'élaboration, l'application et au respect des lois qui protègent les droits des femmes ainsi que l'égalité du genre (Décret $\mathrm{n}^{\circ} 100 / 57$ du 4 avril 2016). Cette direction s'occupe aussi de l'autonomisation de la femme et elle est aussi chargée d'élaborer des rapports sur la mise en œuvre des programmes auxquels le 
Burundi s'est engagé en matière de la promotion de la femme et de l'égalité du genre (Décret $\mathrm{n}^{\circ} 100 / 57$ du 4 avril 2016). Des points focaux genre au niveau de tous les ministères ont été mis en place.

En plus de ces structures de l'administration centrale, le gouvernement du Burundi a créé le Forum National des Femmes par le décret n ${ }^{\circ}$ 100/306 du 21 novembre 2012. Cet organe constitue un cadre d'expression des femmes de toutes les couches sociales sur tous les sujets intéressant leur vie et leurs milieux. Le décret qui définit ses missions lui reconnaît le droit de proposer ses aspirations sur toutes les questions de promotion et de protection des droits de la femme (Décret $n^{\circ}$ 100/026 du 15 février 2017 art 10). La composition de cet organe reflète le souci d'inclusion de toutes les couches de femmes dans l'avancement de leur droit. Les membres sont recrutés au niveau collinaire, communal, provincial et national. Cette représentativité élargie nous parait être un gage de prise en compte des situations variées affectant les droits de la femme (Décret n 100/026 du 15 février 2017 art 4).

Pour opérationnaliser les actions du ministère ayant en charge les questions des droits humains, le gouvernement a initié la création des Centres de Développement Familial et Communautaire (CDFC) (Décret $n^{\circ} 100 / 325$ du 17 décembre 2012). Cet organe décentralisé jusqu'au niveau communal traite de toutes les questions qui rentrent dans le mandat du ministère de tutelle (Décret $\mathrm{n}^{\circ}$ 100/325 du 17 décembre 2012). Aux termes de l'article 6, le texte créant ces centres traite spécifiquement de la promotion des droits des femmes et de l'égalité de genre. Les CDFC sont ainsi habilités à " promouvoir l'égalité des genres en milieu rural et urbain» et à «mettre en application les résolutions et recommandations issues des conférences régionales et mondiales sur la femme et l'égalité des sexes ratifiées par le Burundi» (art 6 al 2 et 4 du Décret n 100/325 du 17 décembre 2012).

Ces diverses initiatives ne peuvent être prises pour produire seulement un effet d'annonce et témoigner d'une sympathie de façade. Des considérations précédentes, il se dessine, au Burundi, une évolution réglementaire et institutionnelle favorable à l'effectivité de la succession foncière de la femme. Mais le constat demeure qu'en traitant cette question, on risque d'être emporté par la passion que par la raison. Si la solution durable à cette question traîne, le retard est beaucoup plus dû au décalage entre l'évolution des mentalités et le rythme d'accélération dans la mise en place des outils institutionnel et réglementaire. Il semble alors que c'est ce temps d'ajustement qui suscite les impatiences alors que dans les outils de programmation à long terme, cette question de succession foncière est prise en compte. 


\subsubsection{Les institutions relevant de la sphère privée}

La défense des droits fonciers successoraux fait partie du champ d'intervention de plusieurs organisations de la société civile (Association pour la Paix et les Droits de l'Homme, Association des femmes juristes du Burundi, Action des Chrétiens pour l'Abolition de la Torture au Burundi). Parfois, ces organisations de la société civile opèrent sous forme de collectif pour accroître la portée de leurs travaux ou pour plus d'efficacité dans l'élaboration des projets qu'ils soumettent à leurs bailleurs (Collectif des Associations et ONG Féminines du Burundi et Fédération Nationale des Associations engagées dans le Domaine de l'Enfance au Burundi). Ces organisations sont pour la plupart des ONG qui interviennent de manière générale sur des thématiques diverses en matière de droit de l'Homme.

Les questions en rapport avec la succession des femmes y sont abordées parfois incidemment. Une littérature abondante sur cette question de succession foncière de la femme inonde la toile. Seulement, certaines des analyses sont beaucoup plus passionnelles que scientifiques; ce qui amène ceux qui le rejettent à qualifier leurs auteurs d'activistes. Les mécanismes d'évaluation de ces travaux que l'on qualifie couramment de consultance consistent en leur validation parfois présidée par leurs commanditaires. Ce sont ceux-ci qui valident les participants à la séance. Cette procédure de validation est peu compatible avec les qualités d'indépendance et d'impartialité qui sont des exigences fondamentales pour le caractère scientifique du travail. Les commanditaires de ces travaux ou les organisations qui financent leurs auteurs poursuivent des objectifs bien précis. Ils définissent des activités à exécuter et choisissent leurs partenaires avec des termes de référence qui rythment avec leur mission. Malgré cette portée scientifique limitée, les travaux de ces ONG gardent leur importance dans le contexte burundais des droits de succession foncière de la femme. Ils permettent de sensibiliser l'opinion publique sur la pertinence de la thématique. Ils rendent compte aussi de la contribution de ces ONG en tant que partenaires accrédités du ministère ayant dans ses attributions les questions de droits de la personne humaine.

\subsubsection{Cadre politique}

La volonté de promouvoir des droits fonciers de la femme sans restriction est incarnée dans plusieurs instruments de planification à long terme. Sans les évoquer tous, l'étude se sert de ceux qui sont les plus illustratifs à savoir le plan national de développement, la politique nationale genre et la lettre foncière pour illustrer la place que ces outils réservent à la femme dans les enjeux fonciers au Burundi. 


\subsubsection{Plan national de développement}

Le Burundi s'est doté d'un Plan National de Développement (PND) qui accorde une place singulière à la femme et à la promotion de l'égalité genre (PND 2018-2027). Les rédacteurs du PND ont fondé sa philosophie en s'inspirant de la vision 2025 qui a été élaborée pour servir de référence pour le développement socio-économique du Burundi pour les générations à venir (Burundi vision 2025). Les planificateurs de la vision 2025 lui avaient assigné un rôle catalyseur dans l'élaboration des politiques et des stratégies pour un développement durable. Cette vision considère la femme comme la force vive du développement et «envisage de mettre en place une politique volontariste de promotion de la femme, notamment à travers l'amélioration de son accès à l'éducation, sa participation politique et aux efforts de développement économique et social du pays » (Burundi vision 2025).

L'un des objectifs stratégiques que le PND propose de mettre en œuvre pour promouvoir une gouvernance axée sur la souveraineté nationale consiste à consolider l'état de droit et les droits humains. Il prévoit l'éradication des inégalités sous toutes leurs formes. Le PND doit être considéré comme un document-cadre qui trace les grandes orientations. Pour opérationnaliser ces objectifs, le PND donne des indications sur la nature des projets à élaborer. Dès lors, l'effectivité de la prépondérance que le PND accorde aux droits humains est sujette à l'existence des acteurs des droits humains ayant des capacités développées pour entreprendre des activités pertinentes en rapport avec la protection de droits de la femme. L'État, en tant qu'acteur central, appuie le renforcement des capacités de ses partenaires. Cet appui peut prendre diverses formes. Il peut s'agir de la mise en place des institutions de soutien technique dans l'élaboration des projets bancables ou encore de soutien financier pour l'exécution des activités prévues.

Cependant les efforts que prennent la mise en place des mesures et l'exécution des décisions prises pour mettre fin aux violations exigent plus qu'un soutien technique ou financier. Une franche collaboration entre les institutions publiques telles que les collectivités locales et les institutions judiciaires avec les intervenants constitue un gage de réussite.

En tant qu'outil de planification, le PND prévoit l'autonomisation des femmes et envisage de leur conférer des appuis pour accroître leur accès aux opportunités économiques (Burundi vision 2025). On voit donc dans la philosophie de cet instrument une large ouverture bienveillante pour la protection des droits de la femme. La démarche qui reste à franchir pour accroitre la protection serait de se doter d'un plan stratégique qui met en évidence les activités et les moyens pour les accomplir ainsi que la définition d'un calendrier d'exécution. 


\subsubsection{La politique nationale genre}

Un consensus existe au sein de la communauté burundaise sur la nécessité de combattre les inégalités qui confinent la femme au rôle de second rang dans la société (le Burundi a ratifié beaucoup de conventions sur les droits des femmes). Le poids démographique de la femme (ISTEBU, Recensement général de 2008), et surtout son rôle de pilier de la cellule familiale imposent un positionnement conséquent sur l'échiquier politique économique, social et culturel. La politique nationale genre dont le Burundi s'est doté en 2012 constitue à cet effet un outil contre les remparts de l'exclusion et des inégalités persistantes visant la femme burundaise (Politique nationale genre [20122025]). La politique nationale genre constitue une volonté manifeste de construire une société qui s'oppose aux considérations attardées et à une culture virile qui fragilisent la femme dans ses droits. Cet idéal de justice construit autour des valeurs d'égalité et d'équité est un ferment pour une société paisible où chaque couche jouit de ses droits fondamentaux (Politique nationale genre, 2012-2025). En dépit de la volonté manifeste d'améliorer les droits des femmes, des obstacles culturels confortent les mentalités rétrogrades qui pérennisent une supériorité supposée ou imaginaire de la gent masculine. La politique nationale genre a été actualisée après neuf ans d'application dans l'optique de tenir compte de nouvelles formes de violations de droit des femmes et des faiblesses révélées par l'évaluation de la politique nationale genre de 2003 (Politique nationale genre, 2012-2025).

\section{Conclusion}

La succession foncière de la femme est soumise à une donnée qui, à défaut de relever de la métaphysique, échappe quand même aux prédictions et aux analyses des juristes les plus chevronnés. Cette donnée, c'est la coutume dont il est impossible de décréter le déclin. La présente étude a démontré la recevabilité du principe d'égalité des droits des femmes et des hommes. La consécration constitutionnelle de l'égalité, l'incorporation de la Déclaration universelle des droits de l'Homme dans la charte africaine des droits de l'Homme et des peuples n'ont apporté que des espoirs d'amélioration des droits de succession foncière de la femme. L'effectivité de ces droits attendra que le déclin possible de la coutume successorale soit mature et que les mentalités burundaises évoluent pour en accepter les effets. L'étude dégage qu'il y a un pas embryonnaire dans la succession des terres en milieux urbains où les enfants se les partagent équitablement et aussi dans le cas d'une descendance féminine (Association pour la paix et les droits de l'Homme). Mais ces avancées sont loin de bouleverser la stabilité de cette coutume savamment entretenue par la position prédominante de la gent masculine dans l'organisation sociale, économique et politique du Burundi. 
Des prémices de l'élargissement de l'accès des femmes à la succession foncière sont disséminées dans les réglementations et dans divers outils de planification à long terme. L'évolution lente de la mentalité de la femme burundaise et le degré de son intégration dans la gestion des affaires publiques constituent des obstacles dans la mise en valeur de ce climat favorable à la succession qui se dessine. Pourtant, le profil numérique de la femme au Burundi illustre son potentiel économique grandissant dans la communauté burundaise (ISTEBU, Recensement général de 2008). En plus d'être majoritaires, elles vivent essentiellement dans les campagnes où elles s'occupent de l'agriculture, activité principale sur laquelle repose l'économie du Burundi. Sur ce, cette étude conclut sur une note d'espoir, car la coutume n'est pas statique. Elle est le reflet de la société, qui elle, est en constante évolution.

\section{References:}

1. Accord d'Arusha pour la Paix et la Réconciliation au Burundi signé le 28 août 2000.

2. Action des Chrétiens pour l'Abolition de la Torture au Burundi, 2014. Créée le 24 novembre 2001 et agréée au ministère de l'intérieur par l'ordonnance ministérielle no 530/266 du 21 février 2003.

3. Association des femmes juristes du Burundi créée en 1995 par l'Ordonnance Ministérielle no 530/245 du 25 juillet 1995.

4. Association pour la Paix et les Droits de l'Homme agréée par l'Ordonnance Ministérielle no 530/689 du 13 août 1998.

5. Association pour la paix et les droits de l'Homme, Droits fonciers des femmes au Burundi le temps de l'action, Rapport alternatif sur la mise en œuvre du pacte international relatif aux droits économiques, sociaux et culturels, Burundi, 2015.

6. Cangelosi Elisabetta \& Sabine Pallas, 2014. Sécuriser les droits fonciers des femmes: enseignements tirés d'expériences au Rwanda et au Burundi, juin 2014, en ligne $<$ https://www.landcoalition.org/sites/default/files/documents/resource s/r-wlr-securing-women-land-rights_web_fr_0.pdf > .

7. Collectif des Associations et ONGs Féminines du Burundi, (CAFOB) créé le 8 avril 1994.

8. Constitution de la République du Burundi promulguée le 7/6/2018.

9. Décret - loi $\mathrm{n}^{\circ} 11 / 008$ du 14 mars 1990 portant ratification du Pacte international relatif aux droits civils et politiques (Burundi).

10. Décret $\mathrm{n}^{\circ} 100 / 72$ du 26 avril 2010 portant adoption de la lettre de politique foncière au Burundi.

11. Décret $\mathrm{n}^{\circ} 100 / 026$ du 15 février 2017 portant révision du décret no 100/306 du 21 novembre 2012 portant création, missions, organisation 
et fonctionnement du forum national des femmes au Burundi, article 4, en ligne: Présidence < http://www.presidence.gov.bi/wpcontent/uploads/2017/03/Decret-026-2017.pdf>.

12. Décret $n^{\circ} 100 / 050 / 97$ du 21 mars 1997 portant nomination des membres du Conseil des Bashingantahe pour l'unité nationale et la réconciliation (Burundi).

13. Décret $n^{\circ} 100 / 325$ du 17 décembre 2012 portant création, structure, missions et fonctionnement des centres de développement familial et communautaire (CDFC) au ministère de la solidarité nationale, des droits de la personne humaine et du genre (Burundi).

14. Décret $n^{\circ} 100 / 57$ du 4 avril 2016, portant missions, organisation et fonctionnement du ministère des droits de la personne humaine des affaires sociales et du genre, en ligne: Présidence < http://www.presidence.gov.bi/wp-content/uploads/2017/04/decret057-2016.pdf>.

15. Décret-loi $n^{\circ}$ 1/001/97 du 3 janvier 1997 fixant l'organisation, la composition et le fonctionnement d'un «Conseil des Bashingantahe pour l'unité nationale et la réconciliation » (Burundi).

16. Décret-loi no 1/006 du 4 avril 1991 portant ratification portant de la Charte africaine des droits de l'Homme et des peuples de 1981.

17. Deslaurier Christine, 2003. Le « bushingantahe » peut-il réconcilier le Burundi ? Politique africaine 2003/4 (No 92), pages 76 à 96.

18. Fédération Nationale des Associations engagées dans le Domaine de l'Enfance au Burundi/FENADEB créée le 08/07/2011 et reconnue officiellement par le Ministère de l'intérieur le 08/08/2011.

19. Gatunange Gervais, 2004. Etude sur les pratiques foncières au Burundi- Essai d'harmonisation -Enquêtes menées dans 10 provinces $d u \quad$ Burundi, en ligne: Lib utexas $<$ https://repositories.lib.utexas.edu/bitstream/handle/2152/4527/3627. pdf;jsessionid=DC999266DBA1 AFC8EAA434FBFB579921?sequen $\mathrm{ce}=1>$.

20. Gatunange Gervais, 2015. Le régime matrimonial de droit commun en droit burundais, Revue burundaise de droit et société Vol. I, no 2 décembre 2015, 35.

21. Gouvernement du Burundi «Communiqué de presse sur la situation sécuritaire », 22 novembre 2011.

22. Index $<$ https://www.indexmundi.com/map/?t=10\&v=21000\&r=af\&l=fr $>$, consultée en 2020.

23. International Crisis Group, 2014. Les terres de la discorde (I):la réforme foncière au Burundi Rapport Afrique no 213 du 12 février 2014, en ligne :

Relief 
$<$ https://reliefweb.int/sites/reliefweb.int/files/resources/213-lesterres-de-la-discorde-i-la-reforme-fonciere-au-burundi.pdf $>$.

24. ISTEEBU, Recensement général de la population et de l'habitat de 2008, en ligne: ISTEEBU

http://www.isteebu.bi/images/rapports/projection\%20de\%201a\%20po pulation\%20bdi\%202008-2030.pdf>.

25. IUCN, 1983. Les principes islamiques pour la conservation de l'environnement naturel, Environmental Policy and Law, paper 20,1983.

26. Journal de presse écrite Iwacu, «Deuxième anniversaire du code foncier : des améliorations et des attentes », 12 août 2013 en ligne : Journal Iwacu < https://www.iwacu-burundi.org/>.

27. Kohlhagen D., 2009. Burundi: La justice en milieu rural: Vers un nouveau code foncier au Burundi, Bujumbura.

28. Kotchikpa G. L., 2017. Droits de propriété foncière, aversion au risque et performance des petits producteurs agricoles, Thèse de doctorat, Université Laval.

29. Laely T.,1992. "Le destin du Bushingantahe : transformations d'une structure locale d'autorité au Burundi», Genève-Afrique, 30 (2), pp. $75-98$.

30. Legitimus http://www.legitimus.ca/static/uploaded/Files/Documents/Rapports/_ Burundi_Rapport-dintegration1.pdf>.

31. Lettre de politique foncière du 15 octobre 2008, en ligne : FAO < http://www.fao.org/fileadmin/user_upload/kagera/resource/TAMP\%2 0Kagera\%20_Lettre_politique_fonciere_nov_08.pdf >.

32. Loi $\mathrm{n}^{\circ} 1 / 016$ du 20 avril 2005 portant organisation de la l'administration communale (abrogée en 2010) (Burundi).

33. Loi $\mathrm{n}^{\circ} 1 / 04$ du 5 janvier 2011portant création de la Commission Nationale Indépendante des Droits l'Homme au Burundi en ligne.

34. Loi $\mathrm{n}^{\circ} 1 / 13$ du 9 août 2011 portant révision du code foncier du Burundi.

35. Loi $\mathrm{n}^{\circ} 1 / 010$ du 7 juin 2018 portant promulgation de la Constitution du Burundi, en ligne: Présidence < https://presidence.gov.bi/2018/07/03/6271/>.

36. Makobero D., 2001. L'institution des Bashingantahe comme moyen de réconciliation », Au cœur de l'Afrique, 1-2.

37. Ministère de la Sécurité publique du Burundi en ligne, 2020 : Ministère de la Sécurité publique <http://www.securitepublique.gov.bi/spip.php?article396>, consulté en janvier 2020. 
38. Ncutinamagara A., 1980. L'évolution des règles de droit successoral coutumier du Burundi et quelques aspects du droit successoral étranger : le cas de la succession ab intestat, Revue juridique du Burundi, no 2.

39. Ntabona A.,1985. "Le concept de l'Umushingantahe et ses implications sur l'éducation de la jeunesse d'aujourd'hui au Burundi », Au Cœur de l'Afrique, 5.

40. PND Burundi, 2018-2027 en ligne: Présidence <http://www.presidence.gov.bi/wp-content/uploads/2018/08/PNDBurundi-2018-2027-Version-Finale.pdf>.

41. Politique nationale genre (2012-2025), en ligne: Présidence, <http://www.presidence.gov.bi/wpcontent/uploads/2017/04/politique _nationale_genre_png_2012-2025.pdf>.

42. Proposition de loi sur les successions, les libéralités et les régimes matrimoniaux de 2004.

43. Rapport parallèle au comité des Nations Unies des droits de l'Homme Burundi, 112e Session (7 Oct 2014 à 31 Oct 2014).

44. RCN Justice \& Démocratie, La justice en milieu rural, Bujumbura, décembre 2009, en ligne: RCN <https://rcnong.be/publications/etudes/decembre-2009-publication-burundi-lajustice-en-milieu-rural/ $>$.

45. Résolution 217 A (III) ayant conduit à l'adoption, le 10 décembre 1948, de la Déclaration universelle des droits de l'Homme à Paris.

46. Rodegem F. M. 1996. "Structures judiciaires traditionnelles au Burundi », Revue juridique de droit écrit et coutumier du Rwanda et du Burundi, 6 (1).

47. Sebudandi, C. \& Ndikumana, V., 2012. À la conquête de la parole La participation des femmes dans la transition démocratique au Burundi, International Alert, Bujumbura. 